\title{
Optimal configuration of a far-infrared radiometer to the study the Arctic winter atmosphere
}

\author{
Laurence Coursol $^{1}$, Quentin Libois ${ }^{2}$, Pierre Gauthier $^{1}$, Jean-Pierre Blanchet ${ }^{1}$ \\ ${ }^{1}$ Université du Québec à Montréal (UQAM), Montréal, Canada \\ ${ }^{2}$ CNRM, Université de Toulouse, Météo-France, CNRS, Toulouse, France
}

\section{Key Points:}

- FIR channels add information for UTLS water vapor compared to standard MIR channels

- IC is used to optimize the channels frequencies and widths of a FIR radiometer

- A high DFS is reached with only a few channels of an optimized FIR radiometer 


\section{Abstract}

Several FIR satellite missions are planned for the next decade, with a special interest for the Arctic region. A theoretical study is performed to help about the design of a FIR radiometer, whose configuration in terms of channels number and frequencies is optimized based on information content analysis. The problem is cast in a context of vertical column experiments (1D) to determine the optimal configuration of a FIR radiometer to study the Arctic polar night. If only observations of the FIR radiometer were assimilated, the results show that for humidity, $90 \%$ of the total information content is obtained with 4 bands whereas for temperature 10 bands are needed. When the FIR measurements are assimilated on top of those from the Advanced Infrared Sounder (AIRS), the former bring in additional information between the surface and $850 \mathrm{hPa}$ and from $550 \mathrm{hPa}$ to $250 \mathrm{hPa}$ for humidity. Moreover, between $400 \mathrm{hPa}$ and $200 \mathrm{hPa}$, the FIR radiometer is better than AIRS at reducing the analysis error variance for humidity. This indicates the potential of FIR observations for improving water vapor analysis in the Arctic.

\section{Introduction}

Since the beginning of meteorological satellites, temperature profiling has been performed with sounders in the infrared (IR) (Wark \& Hilleary, 1969). The state-of-the-art instruments that probe the mid-infrared (MIR) are the Infrared Atmospheric Sounding Interferometer (IASI) (Blumstein et al., 2004), the Atmospheric Infrared Sounder (AIRS) (Aumann et al., 2003) and the Cross-track Infrared Sounder (CrIS) (Bloom, 2001). Those instruments use the $15 \mu \mathrm{m} \mathrm{CO} \mathrm{CO}_{2}$ absorption band to probe atmospheric temperature and the water vapor vibrational band at $6.3 \mu \mathrm{m}$ to retrieve humidity profiles (Rizzi et al., 2002). This humidity profiling capability results from the strong spectral variations of the water vapor absorption in that band.

Water vapor also exhibits an extended rotational absorption band as well as a continuum in the far-infrared (FIR; $15 \mu \mathrm{m}<\lambda<100 \mu \mathrm{m}$ ). This absorption band is broader than the vibrational band, hence there is more energy in this region. Previous studies have pointed out the potential of FIR for atmospheric profiling, particularly in cold regions. First, a direct consequence of the temperature dependence of the Planck function in the radiative transfer equation, emission is shifted to the FIR as the temperature of the scene decreases, offering a greater capability for cold scenes (M. Mlynczak et al., 2007), in contrary to the traditional $6.3 \mu \mathrm{m}$ band quickly loses energy as the scene gets colder (Susskind et al., 2003). Practically, more than half of the radiation is lost to space in the FIR in the polar regions (M. Mlynczak et al., 2007). Second, the water vapor rotational absorption band in the FIR has many absorption lines with larger optical depth than the MIR, which leads to an increased sensitivity to small water vapor variations (Harries et al., 2008). The increased sensitivity is especially important in the upper troposphere, where the water vapor concentration is scarce (Clough et al., 1992). Thus, the FIR region can be valuable for profiling the atmosphere and particularly in the stratosphere and the upper troposphere (Shahabadi \& Huang, 2014).

Despite these acknowledged advantages of the FIR over the MIR for water vapor profiling, no direct spectrally resolved measurements of the atmospheric radiation have been made recently from space. The last measurements in the FIR, up to $25 \mu \mathrm{m}$, were made 40 years ago on two Russian Meteor spacecrafts and 45 years ago by the IRIS (Infrared Interferometer Spectrometer and Radiometer) instruments on the NASA Nimbus III and IV (M. G. Mlynczak et al., 2002), data that has been used to identify changes in spectral outgoing longwave radiation (?, ?). However at the time, the spectral and spatial resolutions of the observations, along with the large noise, prevented from getting much geophysical information out of the data. Since then, low noise liquid helium cooled bolometers operating in the far-IR have been developed, and used for instance in the FarInfrared Spectrometer of the Troposphere (FIRST) instrument (M. G. Mlynczak et al., 
2006). Such systems are however too delicate, massive and expensive to be but on a satellite. This, in combination with the intrinsic higher sensitivity of MIR sensors compared to FIR sensors, explains why no FIR satellite has been flying for decades now.

Only with the recent advent of uncooled systems operating at room temperature that have space missions in the FIR seen a renewal of interest. The series of satellite missions Climate Absolute Radiance and Refractivity Observatory (CLARREO) (Wielicki et al., 2013) is intended to measure spectrally-resolved Earth emission spectrum between $5-50 \mu \mathrm{m}$ with a spectral resolution of $0.5 \mathrm{~cm}^{-1}$ to determine small changes in the spectral outgoing radiation (infrared and reflected solar). The mission aims at detecting decadal changes in climate forcings, responses and feedbacks and to serve for reference intercalibration in space. It thus focuses on global or regional averages and their variations on annual timescales. As a consequence the noise-equivalent temperature difference (NETD), has the requirement to be smaller than $10 \mathrm{~K}$ in the FIR since averaged over a year it will be reduced to $0.01 \mathrm{~K}$ globally. The requirements on the absolute accuracy are on the contrary much more stringent. ESA candidate mision, called FORUM (Far Infrared Outgoing Radiation Understanding and Monitoring) (Palchetti et al., 2016) focuses on studying the forcings and the feedbacks of atmospheric water vapor and of ice clouds on the climate. The recently funded NASA PREFIRE (Polar Radiant Energy in the Far-InfraRed Experiment) CubeSat, to be launched in 2022, intends to measure in the 0-45 $\mu \mathrm{m}$ range to measure spatial and temporal variations in spectral fluxes on hourly to seasonal timescales (L'Ecuyer, 2019). Merrelli and Turner (2012) used the technical characteristics of CLARREO to compare two interferometers for remote sensing of temperature and humidity, with a spectral resolution of $0.5 \mathrm{~cm}^{-1}$, one measuring in the MIR and another measuring in the FIR even though CLARREO was not designed to measure temperature and water vapor. They showed that there is more information content in the FIR compared to the MIR when the noise is equal in both spectral regions. However, if the uncertainty of the actual CLARREO is used for the FIR region, the advantage of the FIR is lost (Shahabadi et al., 2015).

Only a limited selection of channels among those in the water vapor absorption band of interferometers at high resolution is used in data assimilation (Fourrié \& Thépaut, 2003). Supposedly, adjacent bands could thus be merged into larger bands to refine the remote sensing capability. The Arctic was selected since around $60 \%$ of the outgoing longwave radiation is in the FIR region. Also, there is a need for precise water vapor measurements in the troposphere (Müller et al., 2016) and especially in the Arctic (Boullot et al., 2016) and the FIR upwelling spectrum contains a large amount of potential profiling information. The objective of the present study is to design an optimal FIR radiometer to study the Arctic polar night by examining different configurations, noise levels and the tradeoff between spectral resolution and noise level. Thus, this study considers a radiometer in the Arctic region using different filters or a gratings to allow different bandwiths within a spectral region. As in Observing System Simulation Experiments (OSSEs), synthetic measurements are created for different configurations of the FIR radiometer. The radiometric noise of the Far InfraRed Radiometer (FIRR) is used as a baseline to constrain the detector's performance (Libois et al., 2016). The optimal configuration is selected with information content as a metric to lead to the best temperature and humidity analyses. The impact of FIR measurements is also evaluated in terms of their added value when assimilated on top of currently assimilated AIRS data. The experiments are done under the assumption that AIRS and the FIR radiometer are collocated and assimilated in a simple 1D assimilation system.

The paper is organized as follows. Section 2 presents the information content framework, the characteristics of the instrument, and the context of the experiments. Section 3 presents the results of the evaluation of different instrument configurations. Section 4 compares the impact of measurements of the FIR radiometer with that of AIRS measurements. A discussion and conclusions are presented in section 5 . 


\section{Methods}

This section first presents the characteristics of the FIR radiometer and the atmospheric conditions used in this study. Finally, we present the method used to evaluate the information content of measurements which is based on the reduction of analysis error obtained in the context of data assimilation using a numerical weather forecast as an a priori background state.

\subsection{Instrument characteristics}

A synthetic spaceborne FIR radiometer is considered in this study. The goal is not to investigate a particular instrument, but to explore the potential of such a novel instrument, in the framework of the prepartaion of the TICFIRE mission. Nevertheless, the characteristics of this radiometer are based on the FIRR instrument. The characteristics of the optics are fixed (field of view, spatial resolution, F-number, etc), only those of the detector are changed. The detector performance explore a realistic range, although the feasibility study for such performance is left to the industry. The two principal characteristics considered are its number of bands and its noise-equivalent radiance (NER). The bands are adjacent and fully cover the range of $15-100 \mu \mathrm{m}$. The transmittance is one in the bands and zero outside. The bandwidths are set in three different ways hereafter referred as 'equi-energetic', 'constant wavelength' and 'constant wavenumber'. Equienergetic means that each band receives the same amount of energy at the top of the atmosphere (TOA), this is calculated for each atmospheric profile used. This implies that the spectral widths of the bands are not constant. Constant wavelength and constant wavenumber bands means that each bands has the same spectral width in microns or $\mathrm{cm}^{-1}$ respectively. The NER is varied through the experiments, but two specific NER will be highlighted, called baseline NER and target NER. The baseline NER is equal to $0.01 \mathrm{Wm}^{-2} \mathrm{sr}^{-1}$, according to the findings of Libois et al. (2016). Those detector characteristics are consistent with a microbolometer sensor coated with gold black for a integration time of $1 \mathrm{~s}$ (Proulx et al., 2009) The other specific value of NER used, target $\mathrm{NER}$, is equal to $0.002 \mathrm{Wm}^{-2} \mathrm{sr}^{-1}$, which is the expected NER in a few years from now , expected from efforts by the industry, mainly on the electronics and on the analog to digital conversion. Also, band splitting is achievable with a grating or filters. It was chosen to use NER instead of NETD for the radiometric resolution in order to work at the sensor level. This allows to evaluate the gain of changing the radiometric resolution and the spectral width of the bands independently. NER remains constant independently of the instrument spectral configuration, while NETD would change. Since the NER is constant, this results in less energy per band when the bandwidth is reduced. It needs to be noted the correlation between radiometric and spectral resolutions, when the number of bands increases, the signal-to-noise ratio decreases as the energy per band decreases.

Figure 1 shows the NETD for a blackbody at $250 \mathrm{~K}$ for a constant NER of 0.01 $\mathrm{Wm}^{-2} \mathrm{sr}^{-1}$ for an instrument with 10, 15, 20, 25 and 40 equi-energetic bands. The NETD is not constant for the bands of a configuration. This allows to compare this experiment with other studies using NETD. It shows that the NETD, for a configuration with 10 bands, is comparable to the NETD of AIRS, below 0.5 K (Garand et al., 2007), and of MODIS, less than $0.35 \mathrm{~K}$ (Xiong et al., 2008).

\subsection{Atmospheric profiles}

The radiosonde profiles are from the Integrated Global Radiosonde Archive (IGRA) database (http://www.ncdc.noaa.gov/oa/climate/igra/) (Durre et al., 2006). Figure 2 shows the locations of the eight stations where the different vertical profiles were taken. Those stations are the same as in Serreze et al. (2012) and were selected to represent the various atmospheric conditions in the Arctic. It needs to be noted that the Arctic region was chosen, but those results would be similar for the Antarctic region. For each station, 6 profiles were selected randomly from the months of January or February of 2015 


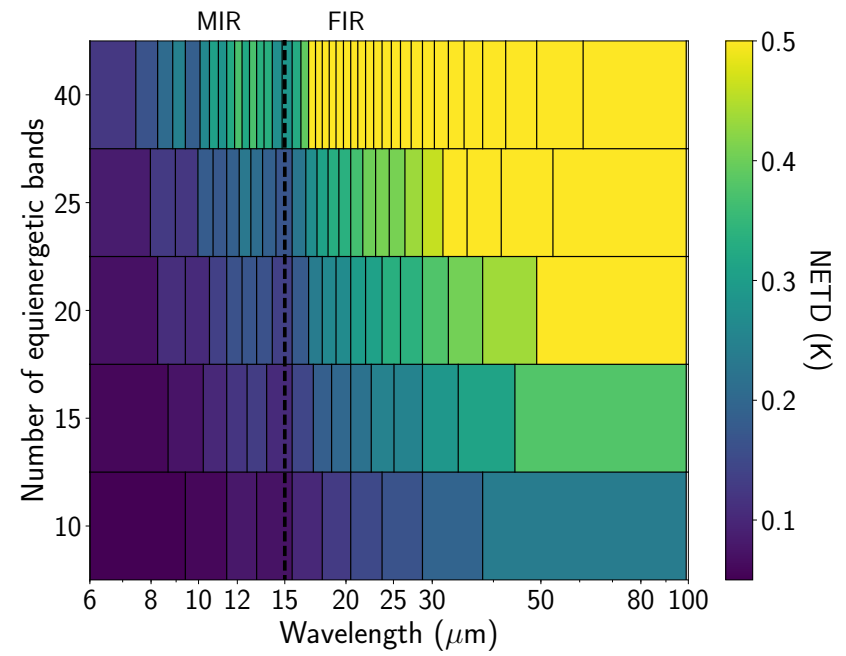

Figure 1. NETD for different configurations of equienergetic bands for a blackbody at $250 \mathrm{~K}$ with a constant NER of $0.01 \mathrm{Wm}^{-2} \mathrm{sr}^{-1}$. The vertical lines represent the widths of equi-energetic bands.

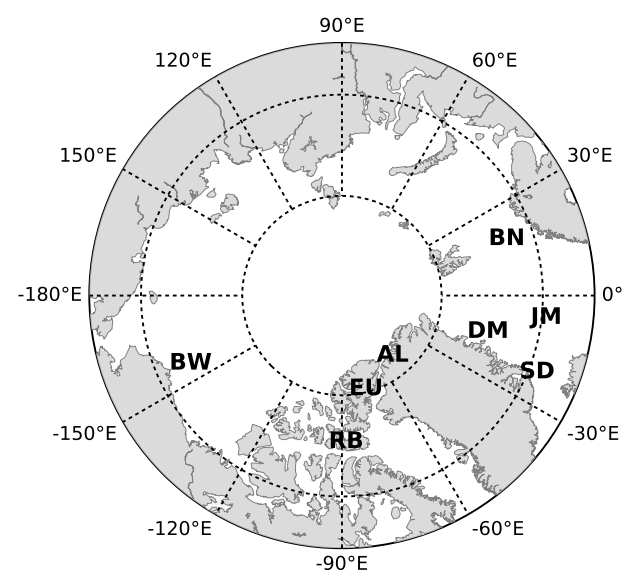

Figure 2. Locations of the eight Arctic stations. The letter codes are: JM- Jan Mayen; BNBjornoya; SD- Scoresbysunde; DM- Danmarkshavn; BW- Barrow, AL- Alert; EU- Eureka; RBResolute Bay.

or 2016 in order to sample Arctic winter conditions. The profiles were truncated at 20 $\mathrm{km}$ altitude. Table 1 shows the vertical resolution of the atmospheric profiles selected. Figure 3 shows the averaged 48 temperature and humidity profiles selected with the red and blue lines respectively and the shaded area of the same color shows the corresponding standard deviation. The natural variability in the profiles seen through the standard deviation can be associated with different meteorological situations. The larger spread near the surface is expected since there is more variability in that region. Also, the peak seen at $4 \mathrm{~km}$ in the standard deviation is due to the averaged water vapor mixing ratio being almost equal to the standard deviation at that point. 
Altitude interval $(\mathrm{km}) \mid$ Vertical resolution $(\mathrm{km})$

\begin{tabular}{l|l}
\hline $0-0.1$ & 0.01 \\
\hline $0.1-1$ & 0.025 \\
\hline $1-3$ & 0.1 \\
\hline $3-5$ & 0.2 \\
\hline $5-8$ & 0.5 \\
\hline $8-12$ & 0.5 \\
\hline $12-20$ & 2
\end{tabular}

Table 1. Vertical resolution of the atmospheric profiles from IGRA

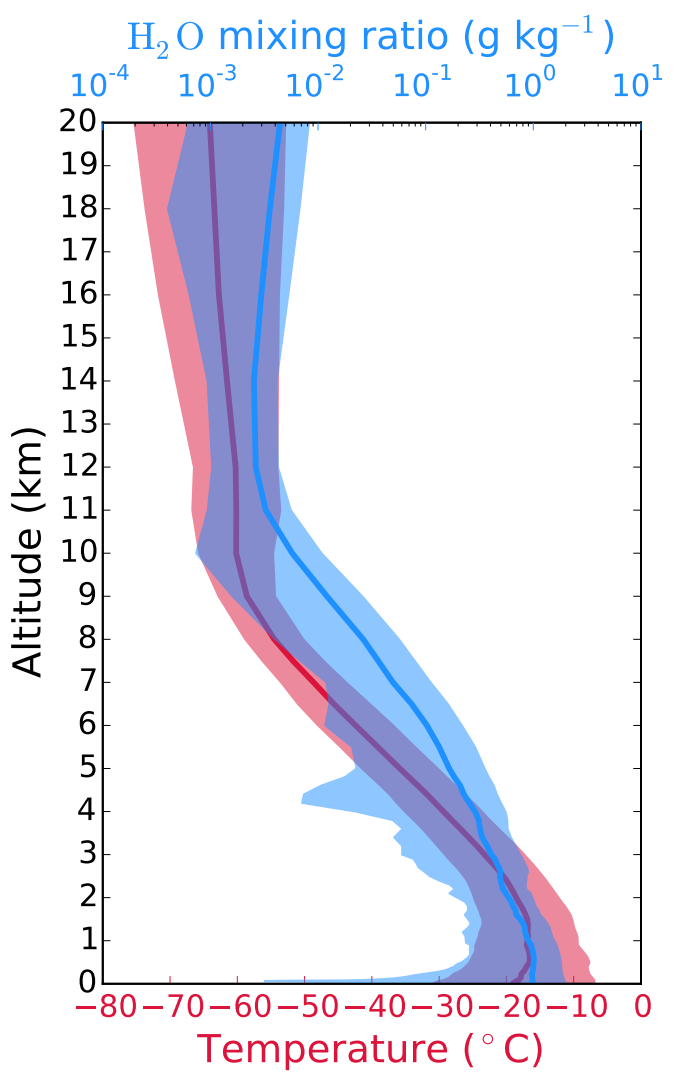

Figure 3. Temperature and humidity profiles averaged for the 48 radiosoundings at eight Arctic stations shown with the red and blue lines respectively. The shaded area shows the standard deviation associated with the variables.

\subsection{Theoretical framework}

In this section, the theoretical framework used is explained. This study is based on linear statistical estimation theory in the context of numerical weather prediction (NWP) (Rodgers, 2000). The different notations, definitions, approximations and data used are described in this section (Lewis et al., 2006). 
The atmospheric state is represented by a vector $\mathbf{x}$ and the satellite radiance measurements at different wavelengths at the TOA are represented by the vector $\mathbf{y}$.

The observation is related to the atmospheric state through the equation

$$
\mathbf{y}=H(\mathbf{x})+\epsilon_{o}
$$

where $H$ is the forward model linking the observation to the atmospheric profile and $\epsilon_{o}$ is the observation error. In this case, the state corresponds to atmospheric profiles of temperature, $\mathbf{T}$, and logarithm of specific humidity $\ln \mathbf{q}$ defined on $\mathrm{k}$ vertical levels on which the model state is defined. The dimension of the model state $\mathbf{x}$ is thus $2 \mathrm{k}$. The ozone and other trace gases are kept constant. The assimilation seeks to correct an a priori estimate of the state of the atmosphere, $\mathbf{x}_{\mathbf{b}}$, also referred to as the background state, using the information contained in the observations. It takes into account the relative accuracies of $\mathbf{x}$ and $\mathbf{y}$ to obtain a minimum variance estimate, $\mathbf{x}_{\mathbf{a}}$, called the analysis.

A linearization of the forward model around the atmospheric profile, $\mathbf{x}_{b}$ is done, which gives, assuming that the radiative-transfer equation is weakly nonlinear near the background state

$$
H(\mathbf{x}) \cong H\left(\mathbf{x}_{b}\right)+\mathbf{H}\left(\mathbf{x}-\mathbf{x}_{b}\right),
$$

where $H\left(\mathbf{x}_{b}\right)$ is the background state in the observations space and $\mathbf{H}=\left.\frac{\partial H(x)}{\partial x}\right|_{x_{b}}$ is the linearized observation operator with respect to $\mathbf{x}$ evaluated at $\mathbf{x}=\mathbf{x}_{b}$, referred to as the Jacobian.

The analysis, $\mathbf{x}_{a}$, which represent the corrected atmospheric state after the observations and the model are taken into consideration, is given by

$$
\mathbf{x}_{a}=\mathbf{x}_{b}+\mathbf{K}\left(\mathbf{y}-\mathbf{H} \mathbf{x}_{b}\right)
$$

with $\mathbf{K}=\mathbf{B} \mathbf{H}^{T}\left(\mathbf{R}+\mathbf{H B H}^{T}\right)^{-1}$ being the gain matrix. $\mathbf{B}$ is the background error covariance matrix and $\mathbf{R}$, the observation error covariance matrix (Rodgers, 2000). The superscript $\mathrm{T}$ and -1 denote respectively the transpose and inverse of a matrix.

\subsubsection{Jacobians}

For each band, the Jacobian indicates how temperature and humidity variations in each band impact the radiance measured at the TOA. The Jacobians were obtained by finite difference with the radiative-transfer model MODTRAN v 5.4 (Berk et al., 2005) by perturbing the background state $\mathbf{x}_{b}$, in this case a temperature and humidity profile. The Jacobians were computed for each band of the FIR radiometer and AIRS. A subset of 142 channels were used for AIRS. It is assumed that the FIR radiometer and AIRS are collocated on a pixel with the same spatial response and taht the lag between the instruments is negligible. More specifically, for the temperature Jacobians, $\mathbf{H}_{T i}$, at the level i, a perturbation of $\pm 0.5 \mathrm{~K}$ was done (Garand et al., 2001). Perturbations of $1 \mathrm{~K}$ have been deemed sufficiently small for this experiment. This gives the variation of radiance seen at the TOA for a variation of $1 \mathrm{~K}$ in the atmospheric profile at each atmospheric level. In the same manner, the humidity Jacobians in logarithm of specific humidity, $\mathrm{s}=\ln \mathrm{q}$, at the level $\mathrm{i}$, were obtained by perturbations of $\pm 0.05 \mathrm{q}$, where $\mathrm{q}$ is the specific humidity and $s=\ln (q)$. As shown with the following equation, in order to obtain a Jacobian with respect to a logarithm, the perturbations are done on the profile in q. Thus by multiplying the difference of perturbations by 10, this results in Jacobians with the units of $\mathrm{Wm}^{-2} \mathrm{sr}^{-1} \log \left(\mathrm{L} \mathrm{L}^{-1}\right)^{-1}$. 


$$
\mathbf{H}_{s, i}=\frac{\partial R}{\partial \ln q}=\frac{\partial q}{\partial \ln q} \frac{\partial R}{\partial q}=q \frac{\partial R}{\partial q}
$$

\subsubsection{Background error covariance matrix}

The matrix $\mathbf{B}$ is the background error covariance matrix associated with the background state $\mathbf{x}_{b}$. Figure 4 represents the $\mathbf{B}$ matrix used for temperature (left) and humidity (right). The $\mathbf{B}$ matrices are the stationary components of the background term of the Environment Canada assimilation system (Buehner et al., 2015). Those matrices were evaluated for a latitude of $79^{\circ} 59^{\prime} 20^{\prime \prime}$, which corresponds to Eureka, Canada for the month of February. The units used are $\mathrm{K}^{2}$ and $\log \left(\mathrm{L} \mathrm{L}^{-1}\right)^{2}$ for temperature and humidity respectively. The cross-terms $\mathbf{B}_{T s}$ and $\mathbf{B}_{s T}$ are considered equal to zero, and thus only the components $\mathbf{B}_{T T}$ and $\mathbf{B}_{s s}$ of the $\mathbf{B}$ matrix are considered. Making this approximation allows to calculate the DFS and analysis error for temperature and humidity separately. In this study, the matrices $\mathbf{B}_{T T}$ and $\mathbf{B}_{s s}$ are kept constant.
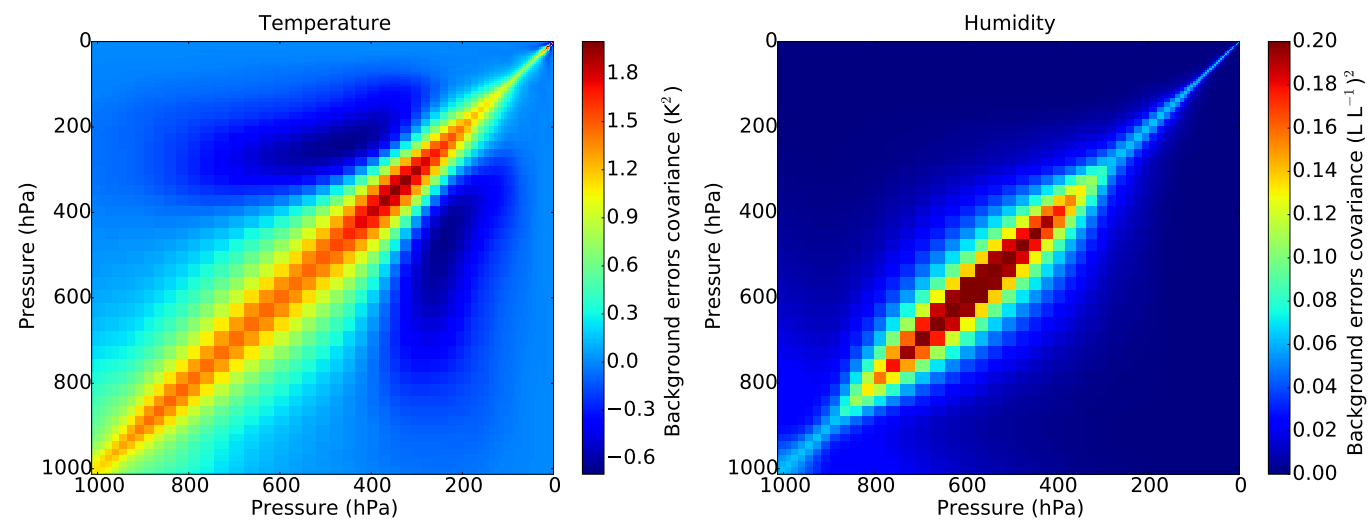

Figure 4. Background error covariances matrices $\mathbf{B}$ for temperature (left) and logarithm specific humidity (right) at a latitude of $79^{\circ} 59^{\prime} 20^{\prime \prime}$ for the month of February

\subsubsection{Observation error covariance matrix}

The matrix $\mathbf{R}$ is the observation error covariance matrix. Normally, the matrix $\mathbf{R}$ takes into consideration the measurement error, the forward-model error, the representativeness error and the error associated with quality control to name a few (Bormann et al., 2010) but for this study, only the measurement error was considered. This approximation was taken to be consistent with previous studies in the FIR region such as Merrelli and Turner (2012), Shahabadi and Huang (2014) and Mertens (2002). They show that, in the thermal IR region, the main contribution to the observation error is the measurement error and also, the interchannel correlation error is small (Garand et al., 2007). The measurement error is assumed to be gaussian and unbiased, assumptions used especially in data assimilation (Rodgers, 2000). Therefore, the matrix $\mathbf{R}$ is assumed to be diagonal with the NER values on the diagonal. The spectral NER for the instrument AIRS was taken from the AIRS website (https://airs.jpl.nasa.gov/index.html) version 5 L1B data. The NER for the synthetic instrument is assumed to be constant for each configuration and each band since it comes from the sensor. 


\subsubsection{Information content}

The impact of measurements is estimated from the analysis error covariance and the degrees of freedom per signal (DFS). The analysis error, assumed here to be unbiased, is $\varepsilon_{a}=\mathbf{x}_{a}-\mathbf{x}_{t}$ where $\mathbf{x}_{t}$ is the true state of the atmosphere. So, $\mathbf{A}=\left\langle\varepsilon_{a} \varepsilon_{a}^{T}\right\rangle$, with $\langle\ldots\rangle$ being the statistical average, is the analysis error covariance matrix and can be shown to be

$$
\mathbf{A}=(\mathbf{I}-\mathbf{K H}) \mathbf{B} .
$$

The reduction of analysis error due to the assimilation of observations is measured by

$$
\operatorname{tr}\left(\mathbf{A B}^{-1}\right)=N-\operatorname{tr}(\mathbf{K H}),
$$

where $\operatorname{tr}(\mathbf{K H})=\operatorname{tr}(\mathbf{H K})$. The gain in information, or the DFS is defined as

$$
\mathrm{DFS}=\operatorname{tr}(\mathbf{H K})
$$

The DFS can then be viewed in two ways, in the observation space and in the model space. In the observation space, the DFS measures the independent degrees of freedom measured by the observations and take into account redundancy. In the model space, it measures the reduction of analysis error with respect to the background error.

It is an evaluation technique based on the relative errors between the observations and the prior information (Purser \& Huang, 1993). It has also been used to quantify the added value of a new set of observations by comparison with other types of measurements and also on top of measurements already assimilated (McNally et al., 2006; Lupu et al., 2011)

Thus, the analysis error variance matrix $\mathbf{A}$ and the DFS depend on the background error covariance matrix $\mathbf{B}$, the observation error covariance matrix $\mathbf{R}$ and the Jacobian matrix $\mathbf{H}$. The DFS will be used as a metric for obtaining the optimal configuration of the FIR radiometer and to discuss the trade-off between spectral resolution and noise level. The analysis error variance matrix will be used to see the vertical impact when the FIR radiometer is assimilated. Those calculations for the DFS and analysis error variance were done for the 48 atmospheric profiles individually, and are then averaged. The calculations for 48 atmospheric profiles show the added value on average and also its variability for the different possible atmospheric situations in the Arctic. The standard deviation spread for the DFS and the analysis error variance will be shown in figures 7 and 10 respectively.

\section{Evaluation of configurations}

\subsection{Optimization under constraints}

In this section, the DFS is used to discuss the trade-off between spectral resolution and noise level.

Figure 5 shows the total DFS for temperature of different configurations for a synthetic FIR radiometer with constant wavenumber configuration when the NER error level varies between 0.0003 and $0.02 \mathrm{Wm}^{-2} \mathrm{sr}^{-1}$ and a spectral range of 15 to $100 \mu \mathrm{m}$. The total number of bands varies between 1 and 200 bands. The color represents the value of the DFS for this configuration. Hence, this figure shows that for a fixed NER, the DFS increases and then decreases as the number of bands increases. This figure can be useful when there are technological constraints for example. If the NER is imposed by the available technology, taking a horizontal line on the top panel of figure 5 highlights the 


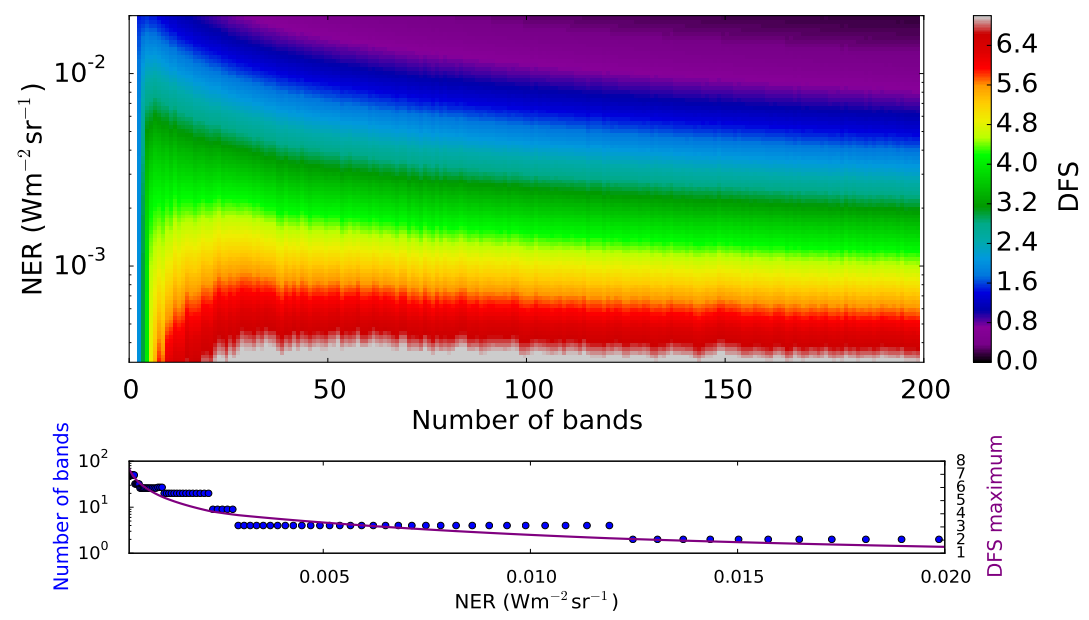

Figure 5. Top panel: The averaged total DFS is shown for variations of the NER level (yaxis) and variations of the total number of bands for temperature of the constant wavenumber bands configuration. The DFS for each configuration is shown with the colorbar. Bottom panel: The number of bands that maximizes DFS as a function of the NER (blue dots) and the DFS maximum as a function of the NER (purple line).

available spectral configurations. The maximum DFS is not with smaller bands (right side of the figure) but always with a configuration which has less than 50 bands. This is due to the constant NER. By having smaller bands, the amount of energy per band decreases and hence the signal-to-noise ratio decreases. This is also shown in the bottom panel, which shows with blue dots the number of bands of the configuration with the maximal DFS for a variation of the NER. This shows that having more bands is not always the best configuration, since the DFS is not increasing as the number of bands increases. The number of bands with the peak DFS gets larger as the NER decreases. Another interesting way to analyze this map is by having a constraint on the number of bands an instrument can have. A radiometer can be operated with a filter-wheel and, as the number of bands increases, the rate of repetition decreases and also the cost increases.

Figure 6 is similar to figure 5 but for humidity, but was cut off at 100 bands to better see the shift in the DFS peak. It has a lot more variability in the DFS for an horizontal line compared to temperature. This variability is partly due to spectral features of transmittance. For humidity, the maximum DFS is always obtained with a configuration which has less than 55 bands.

\subsection{Maximisation of the total DFS}

In this section, the DFS is used to find an optimal configuration for the FIR radiometer considered in this study. Three different splitting of the bands are considered, equi-energetic, constant wavelength bands and constant wavenumber bands. The instrument will be split between 1 and 250 bands.

Considering those three configurations, figure 7 shows the total DFS averaged over the 48 profiles for temperature and humidity. Two values of the NER were used for the level of error, namely $0.01 \mathrm{Wm}^{-2} \mathrm{sr}^{-1}$ and $0.002 \mathrm{Wm}^{-2} \mathrm{sr}^{-1}$. To show the variability, the standard deviation is shown with the shaded area. Note that several papers have investigated the channel selection and information content of AIRS (e.g. Fourrié and Thépaut 


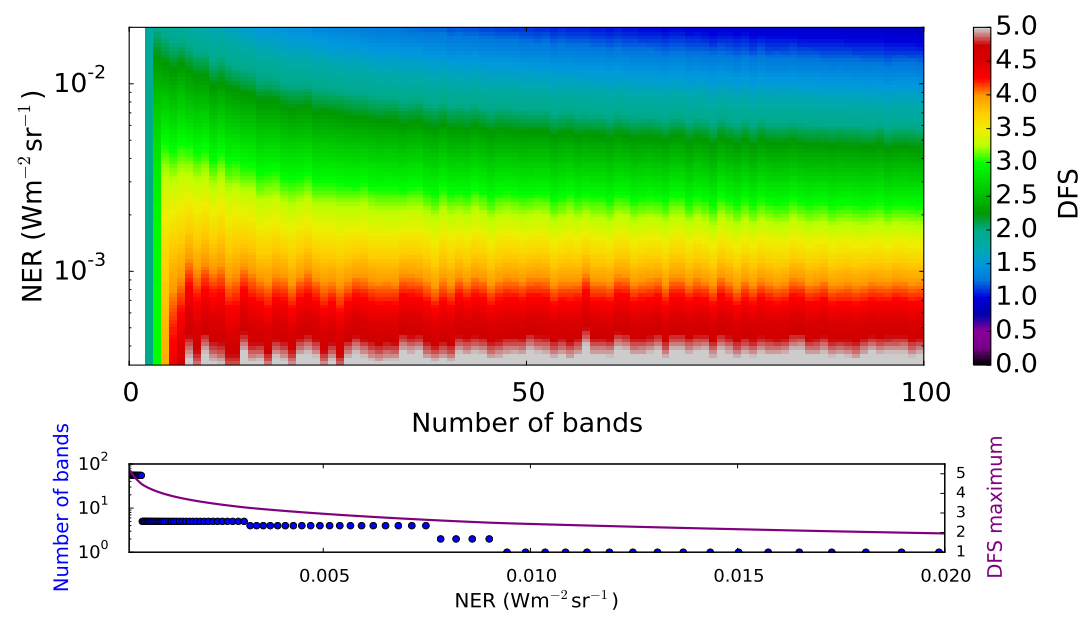

Figure 6. Top panel: The averaged total DFS is shown for variations of the NER level (yaxis) and variations of the total number of bands for humidity of the constant wavenumber bands. The DFS for each configuration is shown with the colorbar. Bottom panel: The number of bands that mazimizes DFS as a function of the NER (blue dots) and the DFS maximum as a function of the NER (purple line).

(2003); Divakarla et al. (2006); Garand et al. (2007)). However, different B and R matrices were used, which prevents direct comparison with this previous work.

For temperature, for the three cases, as the number of bands increases the total DFS decreases. This is due to the constant NER, which results in less energy when the bandwidth is reduced. For the configuration having constant wavelength bands, the peak in DFS is reached with more bands compared to the other configurations. The bands being too wide near $15 \mu \mathrm{m}$, there is not enough resolution to capture the variation in the transmittance in this part of the spectrum. Table 2 shows the maximum DFS for each configuration with the corresponding number of bands for both temperature and humidity for the target noise. When the NER is reduced, there is a large increase in the DFS as expected since it means that the measurements are more accurate. For example, there is an increase by a factor of 2.00 in the DFS for the constant wavenumber band configuration when the NER goes from $0.01 \mathrm{Wm}^{-2} \mathrm{sr}^{-1}$ to $0.002 \mathrm{Wm}^{-2} \mathrm{sr}^{-1}$. For the different configurations, the standard deviation varies between 0.209 to 0.247 which is less than for AIRS which is equal to 0.513 for temperature. The highest DFS is with the constant wavenumber band configuration with 22 bands for the NER level of $0.002 \mathrm{Wm}^{-2} \mathrm{sr}^{-1}$. This is the configuration that will be used for temperature for the remainder of this study. The individual DFS of the optimal configuration of the 22 constant wavenumber bands is $20 \%$ smaller than AIRS. However, when those bands are assimilated on top of AIRS, (table 2), the value of the DFS increases by $13.2 \%$ compared to when AIRS is assimilated alone. This means that even after the information in the thermal IR is assimilated, there is still value in assimilating data in the FIR.

For humidity, the highest DFS is also with the constant wavenumber bands configuration with now 7 bands and a total DFS of 3.594 for the NER error of $0.002 \mathrm{Wm}^{-2} \mathrm{sr}^{-1}$. For the remainder of the study, this configuration will be considered as the optimal configuration . Similarly to temperature, there is more variability for the constant wavelength configuration. For humidity, the standard deviation is larger compared to temperature, i.e. it varies between 0.318 and 0.354 depending on the configuration while it is 0.666 for AIRS. Also, when compared individually to AIRS (table 2), the DFS for the optimal configuration with 7 constant wavenumber bands is smaller than the DFS of AIRS by $14 \%$. 

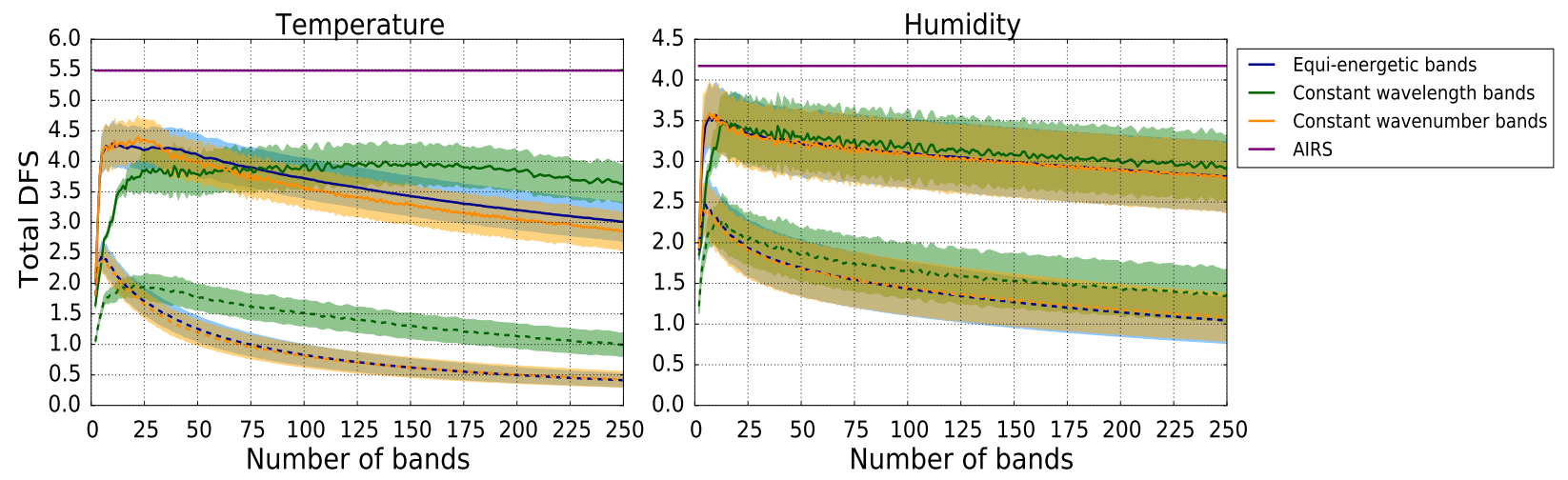

Figure 7. The averaged total DFS as a function of the total number of bands for three configurations which are equi-energetic bands (blue line), constant bandwidths in terms of wavelength (green line) and wavenumber (orange line) for temperature (left) and humidity (right). The dashed lines are for a NER of $0.01 \mathrm{Wm}^{-2} \mathrm{sr}^{-1}$ whereas the full lines are for the target NER of $0.002 \mathrm{Wm}^{-2} \mathrm{sr}^{-1}$. The shaded area represent the standard deviation of the 48 atmospheric profiles which are shown for all configurations except for AIRS. The standard deviation of AIRS is equal to 0.53 and 0.67 for temperature and humidity respectively. The purple line represents the averaged total DFS of AIRS for the 48 atmospheric profiles.

When those 7 bands in the FIR are assimilated on top of AIRS (table 2), the DFS increases by $11.5 \%$, compared to assimilating only AIRS, which shows that measurements in the FIR add information when assimilated on top of AIRS data.

\subsection{Selection of the bands with most information}

Considering a fixed number of bands for the FIR radiometer, following Rabier et al. (2002), we now evaluate the DFS sequentially. First, for each atmospheric profile, the DFS is calculated for each band of the configuration and the one that maximises the DFS is selected. It is shown as the first position in figure 8. The next band selected is the one that, when added to the previous one, adds the largest information content. This process is done until all the bands are selected. Each new band thus optimally increases the DFS. This type of calculation was also done for ice cloud properties for AIRS by Chang et al. (2017). This selection was done for each of the 48 atmospheric profiles and for both temperature and humidity. Thus, figure 8 shows the frequency each band is selected at each position for the optimal configuration of the FIR radiometer with 22 constant wavenumber bands for temperature. It shows that $50 \%$ of the time, the first band selected correspond to the first band (dark orange) in the splitting with the bandwidth 15.02-15.62 $\mu \mathrm{m}$. In first and second position, the bands selected are always between $15.02 \mu \mathrm{m}$ and $20.58 \mu \mathrm{m}$. Also, in the last position, the last band (dark pink) with bandwidth 79.53$100 \mu \mathrm{m}$ is selected $66.67 \%$ of the times whereas the second to last band in the splitting (bandwith $66.03-79.53 \mu \mathrm{m}$ ) is selected $33.33 \%$ of the time.

Similarly for humidity, figure 9 shows the order of selection of the 7 constant wavenumber bands with respect to humidity through the atmospheric profiles. It shows that the first band selected is $58.3 \%$ of the time the third band (mint green) which has boundaries of $19.83-23.62 \mu \mathrm{m}$ whereas the second band (yellow) is selected $22.92 \%$ of the times. For the second band selected, $62.5 \%$ of the time, it is the 5 th band (dark blue) that is selected. The last band selected is always the first band (orange) which has boundaries between $15.02-17.09 \mu \mathrm{m}$. 
Number of bands DFS Analysis error variance

\begin{tabular}{llll}
\hline \multicolumn{3}{c}{ Temperature } \\
\hline Constant wavenumber bands & 22 & 4.399 & 12.64 \\
Constant wavelength bands & 138 & 3.996 & - \\
Equi-energetic bands & 10 & 4.294 & - \\
AIRS & - & 5.488 & 10.58 \\
Background error & - & - & 19.62 \\
AIRS + constant wavenumber bands & 22 & 6.213 & 9.97 \\
\hline \multicolumn{4}{l}{} \\
\hline Constant wavenumber bands & Humidity & \\
Constant wavelength bands & 7 & 3.594 & 1.09 \\
Equi-energetic bands & 15 & 3.482 & - \\
AIRS & 10 & 3.569 & - \\
Background error & - & 4.173 & 1.03 \\
AIRS + constant wavenumber bands & - & - & 1.96 \\
Table & & 4.714 & 0.95
\end{tabular}

Table 2. Total averaged DFS for a NER of $0.002 \mathrm{Wm}^{-2} \mathrm{sr}^{-1}$ and analysis error variance. The units for the analysis error variance is $K^{2}$ and $\log \left(L L^{-1}\right)^{2}$ for temperature and humidity respectively

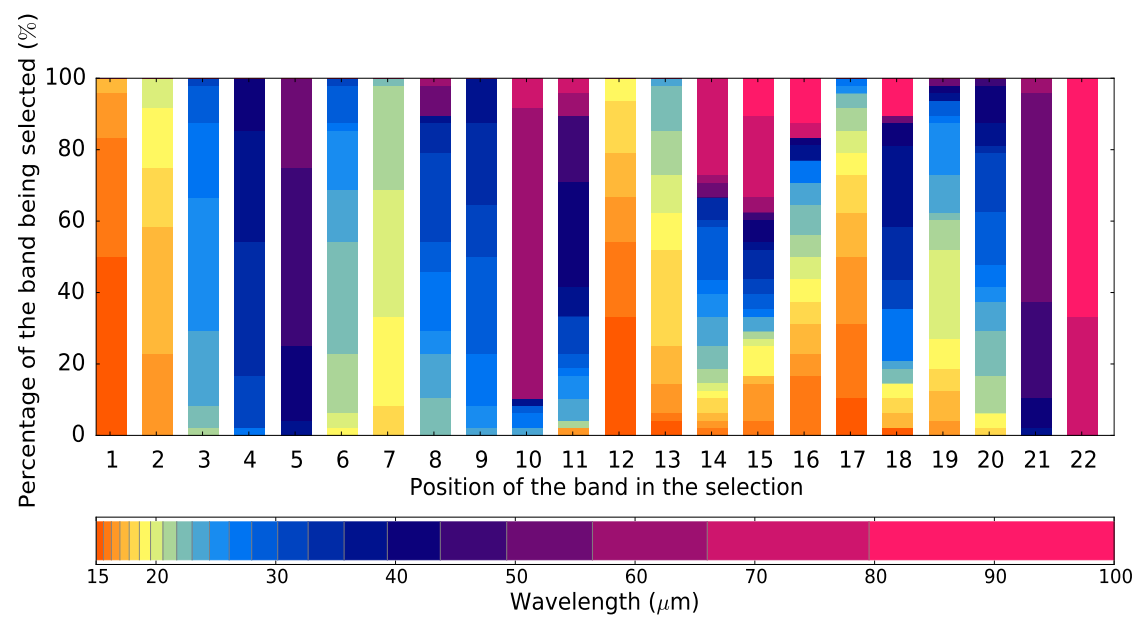

Figure 8. Probability of each band to be selected at each position for the 22 constant wavenumber bands with respect to temperature.

\section{Analysis error}

The impact of the observations can be seen with the analysis error variance since it provides information about the vertical distribution. The DFS previously discussed gives information integrated through the profile. Equation 2.3 is used to obtain the analysis error variance. To show the added value of the FIR radiometer when other types of instruments are assimilated, the instrument AIRS is considered.

Figure 10 shows the analysis error variance profile for temperature and humidity when the optimal FIR radiometer and AIRS are assimilated. The dark green curve represents the background error, the dark blue curve is the average over the 48 atmospheric profiles for the FIR radiometer whereas the shaded area represents the standard deviation associated with the variability obtained through the different profiles. The dark 


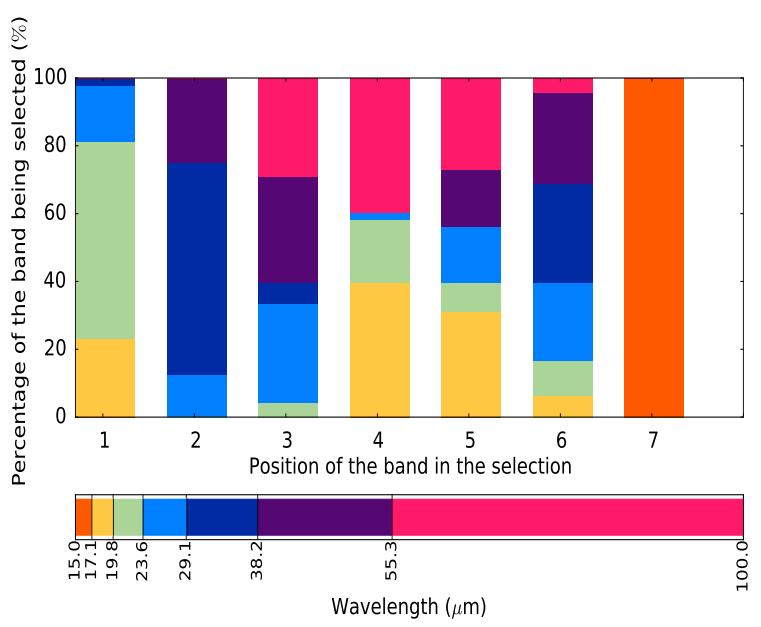

Figure 9. Probability of each band to be selected at each position for the 7 constant wavenumber bands with respect to humidity.

pink curve represents the averaged analysis error variance for AIRS whereas the peach curve shows when the FIR radiometer is assimilated with AIRS. For temperature, AIRS is better at reducing the error compared to the FIR radiometer except at $300 \mathrm{hPa}$. It is also at this height that the gain in assimilating the FIR radiometer on top of AIRS is seen. Also, the sum of the analysis error variance through the profile is shown in table 2, which allows to see the impact of the observations in the model space. Hence, assimilating the FIR radiometer after AIRS allows to reduces by $5.65 \%$ the analysis error variance. The main gain of the FIR radiometer is with respect to humidity. The FIR radiometer is better at reducing the error in the upper atmosphere, between $400 \mathrm{hPa}$ and $200 \mathrm{hPa}$, than AIRS. When the 7 bands of the FIR radiometer are assimilated on top of AIRS, there is a non negligible gain near the surface (between the surface and $850 \mathrm{hPa}$ ) and in the upper part of the atmosphere (between $400 \mathrm{hPa}$ and $200 \mathrm{hPa}$ ). The gain near the surface is due to the FIR radiometer being sensitive to the temperature inversion layer. For the different profiles, there is some variability in the atmospheric conditions which is seen with the standard deviation, especially between the surface and $600 \mathrm{hPa}$ which is expected. In the same way, with table 2 , it shows that assimilating the FIR radiometer after AIRS allows to reduce by $12.84 \%$ the analysis error variance for humidity. Mertens (2002) did a similar study on the ability of the FIR to improve water vapor retrievals. The conclusions are similar in the sense that both studies find that the main reduction in the analysis error variance is between $1000 \mathrm{hPa}$ and $100 \mathrm{hPa}$ and that there is a gain in using both the MIR and the FIR.

To show the impact of each individual band on the analysis error variance profile, the humidity Jacobians of the FIR radiometer (left panels) and the analysis error variance associated with each of these bands when assimilated sequentially (right panels) is shown in figure 11 for two specific atmospheric cases with (top panel) and without (bottom panel) a temperature inversion. The colors of the bands of the Jacobians are associated with the colorbar. The top left panel of figure 11 shows the signature of two effects: the greenhouse effect and the presence of a temperature inversion layer. The negative peak is due to the greenhouse effect of water vapor. Increasing the humidity tends to reduce emission of radiance by masking the lower warmer layers. The positive part near the surface is due to an inversion. Increasing humidity elevates the effective emmision altitude, where the atmosphere is warmer due to the inversion (?, ?). Moreover, as the wavelength of the band increases, the peak's height increases from around $600 \mathrm{hPa}$ 


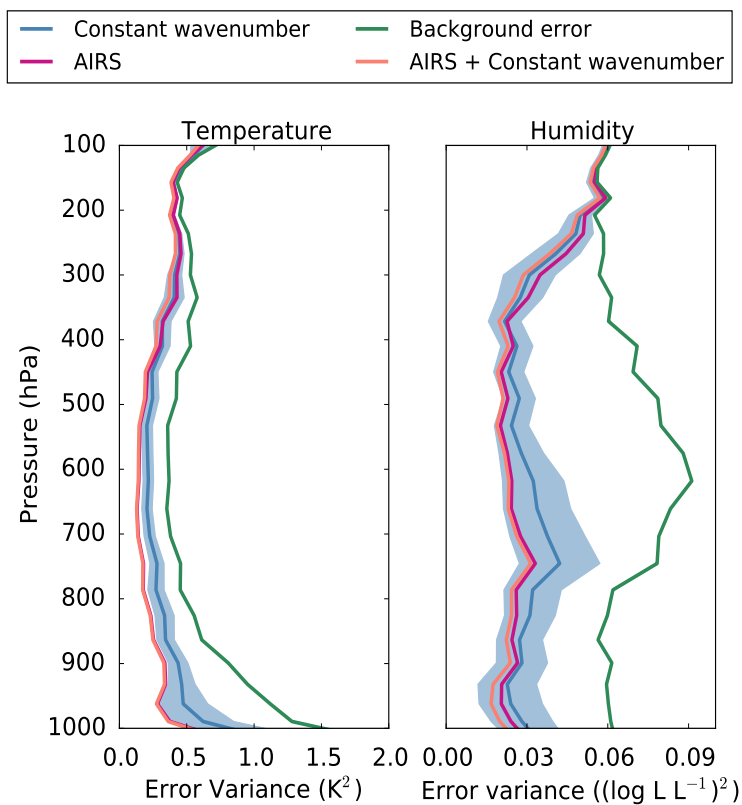

Figure 10. Analysis error variance profile for temperature (left) and humidity (right) for the optimized FIR radiometer. The green curve represents the background error $\mathbf{B}$, the dark blue curve the average on the 48 atmospheric profile whereas the shaded area represents its associated standard deviation. The dark pink line is the analysis error variance of AIRS whereas the peach line represents when the optimized FIR radiometer is assimilated after AIRS.

to $350 \mathrm{hPa}$. The top right panel of figure 11 shows, as in figure 10, the analysis error variance for the background error, when all the bands of the FIR radiometer and AIRS are assimilated separetely which is represented by the dark green, blue and dark pink lines respectively. To show the impact on the analysis error of each band of the FIR radiometer, bands were sequentially assimilated and the analysis error variance was calculated after each new band of the FIR radiometer was assimilated. The order of assimilation of each band is the same as in section 3.3. It shows that the first band assimilated, the pale green one, reduces the error between $800 \mathrm{hPa}$ and $300 \mathrm{hPa}$, which is where the Jacobian's peak is. The second band, navy blue one, has a higher Jacobian's peak (at around $400 \mathrm{hPa}$ ) and it is mainly where the analysis is reduced, between $650 \mathrm{hPa}$ and $200 \mathrm{hPa}$. The next two bands assimilated, yellow and purple lines respectively, are also shown in the figure. The reduction of the analysis error near the surface is mainly due to the yellow band (17.1- $19.8 \mu \mathrm{m}$ ), which is interesting since it allows to restrain the uncertainty in the inversion layer. This illustrates the complementarity of these bands to obtain the best analysis over the whole vertical extent. Compared to AIRS (dark pink line), the FIR radiometer is better at reducing the error between $350 \mathrm{hPa}$ and $250 \mathrm{hPa}$. To show the impact of the temperature inversion, the bottom panels of figure 11 shows the Jacobians and the analysis error variances for an atmospheric case without a temperature inversion. The left panel shows that the Jacobians are more spread out and sample the atmosphere from near the surface up to $300 \mathrm{hPa}$. Also, compared to the other case, there is no positive peak in the Jacobians which is expected since it is due to a temperature inversion layer. For the analysis error variance (bottom right panel), with the Jacobians peak being lower, the analysis error variance is less reduced compared to the case with an inversion. Even though, the FIR radiometer reduces more the analysis error variance than AIRS between $350 \mathrm{hPa}$ and $200 \mathrm{hPa}$. Also, near the surface, the FIR radiometer is better at reducing the analysis error variance when there is an inversion. 


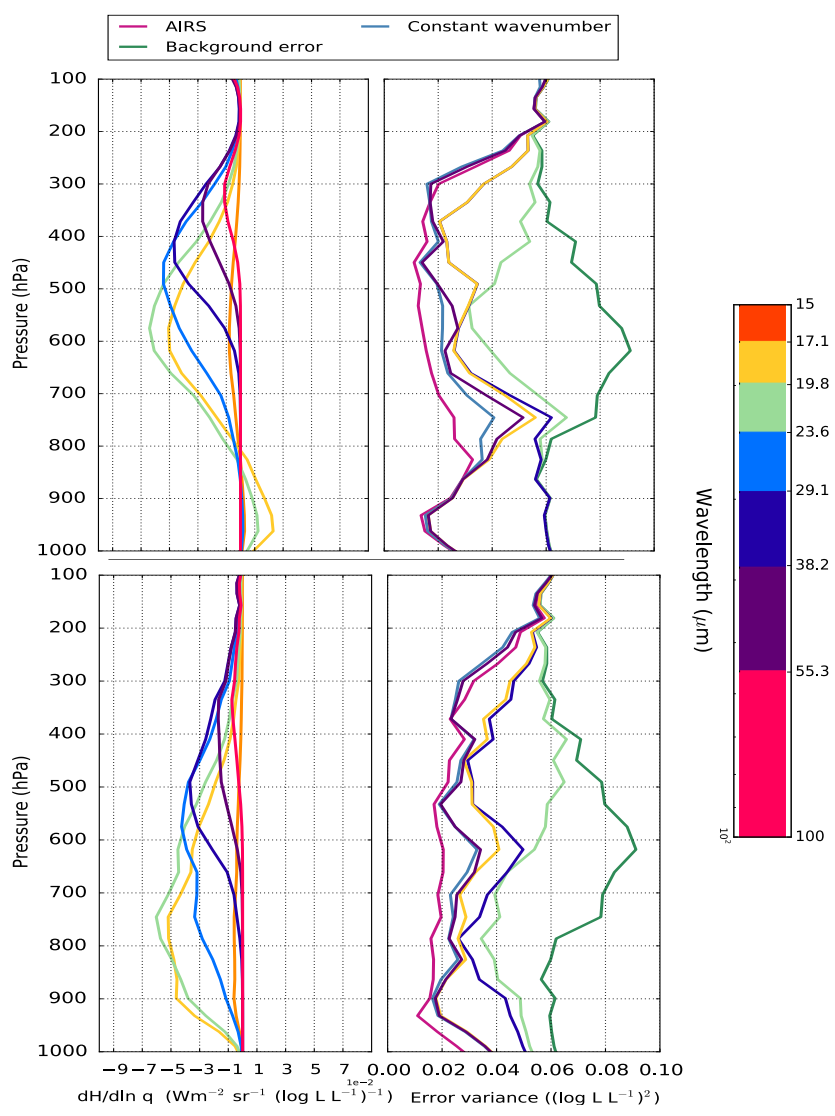

Figure 11. Left panels show the humidity Jacobians for the FIR radiometer associated with two atmospheric cases, which are with (top panel) and without (bottom panel) a temperature inversion. The different colors are the 7 associated bands of this configuration shown by the colorbar which is valid for both panels. The right panel show the analysis error variance for the two cases. The curves from right to left are the background error, when the bands of the FIR radiometer are assimilated one at the time from one band to four (light green, dark navy, yellow and purple), when all the 7 bands are assimilated and AIRS is assimilated.

\section{Conclusions}

The objective of the present study is to design an optimal FIR radiometer to study the Arctic polar night by examining different configurations, noise levels and the tradeoff between spectral resolution and noise level. This was investigated through an information content analysis based on optimal estimation method. The optimal configuration for the synthetic FIR radiometer is with 22 constant wavenumber bands for temperature and 7 constant wavenumber bands for humidity. It was shown that too many bands with a large noise do not give enough information on the atmosphere. With a few bands, it was possible to get a DFS similar to AIRS when compared individually. Given that AIRS provides information also on both temperature and humidity, the impact of assimilating FIR measurements on top of AIRS data was evaluated by the reduction in analysis error variance. With respect to temperature, there is a small impact in assimilating the FIR radiometer measurements over AIRS between 400 and $250 \mathrm{hPa}$. On the contrary, for humidity, there is a non negligible gain near the surface (between the surface and $850 \mathrm{hPa}$ ) and in the upper part of the atmosphere (between $400 \mathrm{hPa}$ and 200 
$\mathrm{hPa}$ ). Something else that is worth noting is that between $400 \mathrm{hPa}$ and $200 \mathrm{hPa}$, taken individually, the FIR radiometer is better at reducing the humidity analysis error variance than AIRS.

Measurements in the FIR are unlikely to be assimilated in the next few years in NWP systems, however the results shown in this paper highlight the potential of this new type of observations which may become available in the next decade. It is non negligible to get results similar to AIRS in reducing the analysis error for humidity with only 7 bands compared to a subset of 142 bands from AIRS. FIR measurements could be used in regions where there is still large uncertainties in water vapor retrieval or assimilation. It was shown to be useful for retrieval of water vapor in the $400 \mathrm{hPa}$ to $200 \mathrm{hPa}$ region for the Arctic, but FIR radiometry can be useful in other regions as well.

The results presented here, are based on a 1D assimilation of two collocated instruments. Another interesting aspect of this study is the method, which facillitates testing rapidly multiple configurations of an instrument. Also, it allowed to compare the relative impact of measurements in the FIR and the MIR. However, there are limitations to this approach that need to be kept in mind. Because a satellite does provides measurements over the whole globe, it would ibe mportant to examine the impact one could expect in other regions such as the Tropics for instance. Finally, complex Observing Simulated Systems Experiments (OSSEs) would be needed to evaluate the global impact in a context including all observations currently assimilated.

\section{Acknowledgments}

This research has been funded in part by the Canadian Space Agency (CSA) through the FAST program, the Grants and Contribution program of Environment and Climate Change Canada (ECCC) and the Natural Sciences and Engineering Research Council of Canada (NSERC) Discovery Grant program. The radiative transfer simulations were all performed with MODTRAN v. 5.4 (http://modtran.spectral.com). The atmospheric profiles used are from 48 radiosondes taken from the IGRA database (ftp://ftp.ncdc.noaa.gov/pub/data/igra/). These simulations and the codes used to generate the figures are available from Laurence Coursol.

\section{References}

Aumann, H. H., Chahine, M. T., Gautier, C., Goldberg, M. D., Kalnay, E., McMillin, L. M., ... others (2003). Airs/amsu/hsb on the aqua mission: Design, science objectives, data products, and processing systems. IEEE Transactions on Geoscience and Remote Sensing, 41(2), 253-264.

Berk, A., Anderson, G. P., Acharya, P. K., Bernstein, L. S., Muratov, L., Lee, J., ... others (2005). Modtran 5: a reformulated atmospheric band model with auxiliary species and practical multiple scattering options: update. In Proc. of spie vol (Vol. 5806, p. 663).

Bloom, H. (2001). The cross-track infrared sounder (cris): A sensor for operational meterological remote sensing. In Fourier transform spectroscopy (p. JTuB1).

Blumstein, D., Chalon, G., Carlier, T., Buil, C., Hebert, P., Maciaszek, T., ... others (2004). Iasi instrument: Technical overview and measured performances. In Proc. of spie vol (Vol. 5543, p. 197).

Bormann, N., Collard, A., \& Bauer, P. (2010). Estimates of spatial and interchannel observation-error characteristics for current sounder radiances for numerical weather prediction. ii: Application to airs and iasi data. Quarterly Journal of the Royal Meteorological Society, 136(649), 1051-1063.

Boullot, N., Rabier, F., Langland, R., Gelaro, R., Cardinali, C., Guidard, V., ... Doerenbecher, A. (2016). Observation impact over the southern polar area during the concordiasi field campaign. Quarterly Journal of the Royal Meteorological Society, 142(695), 597-610. 
Buehner, M., McTaggart-Cowan, R., Beaulne, A., Charette, C., Garand, L., Heilliette, S., ... others (2015). Implementation of deterministic weather forecasting systems based on ensemble-variational data assimilation at environment canada. part i: The global system. $\quad$ Monthly Weather Review, 143(7), 25322559 .

Chang, K.-W., L'Ecuyer, T. S., Kahn, B. H., \& Natraj, V. (2017). Information content of visible and midinfrared radiances for retrieving tropical ice cloud properties. Journal of Geophysical Research: Atmospheres, 122(9), 4944-4966.

Clough, S. A., Iacono, M. J., \& Moncet, J.-L. (1992). Line-by-line calculations of atmospheric fluxes and cooling rates: Application to water vapor. Journal of Geophysical Research: Atmospheres, 97(D14), 15761-15785.

Divakarla, M. G., Barnet, C. D., Goldberg, M. D., McMillin, L. M., Maddy, E., Wolf, W., ... Liu, X. (2006). Validation of atmospheric infrared sounder temperature and water vapor retrievals with matched radiosonde measurements and forecasts. Journal of Geophysical Research: Atmospheres, 111(D9).

Durre, I., Vose, R. S., \& Wuertz, D. B. (2006). Overview of the integrated global radiosonde archive. Journal of Climate, 19(1), 53-68.

Fourrié, N., \& Thépaut, J.-n. (2003). Evaluation of the airs near-real-time channel selection for application to numerical weather prediction. Quarterly Journal of the Royal Meteorological Society, 129(592), 2425-2439.

Garand, L., Heilliette, S., \& Buehner, M. (2007). Interchannel error correlation associated with airs radiance observations: Inference and impact in data assimilation. Journal of applied meteorology and climatology, 46(6), 714-725.

Garand, L., Turner, D., Larocque, M., Bates, J., Boukabara, S., Brunel, P., ... others (2001). Radiance and jacobian intercomparison of radiative transfer models applied to hirs and amsu channels. Journal of Geophysical Research: Atmospheres, 106 (D20), 24017-24031.

Harries, J., Carli, B., Rizzi, R., Serio, C., Mlynczak, M., Palchetti, L., .. Masiello, G. (2008). The far-infrared earth. Reviews of Geophysics, 46(4).

L'Ecuyer, T. (2019). The polar radiant energy in the far infrared experiment (prefire): A far infrared mission to improve estimates of the polar energy budgets. In Geophysical research abstracts (Vol. 21).

Lewis, J. M., Lakshmivarahan, S., \& Dhall, S. (2006). Dynamic data assimilation: a least squares approach (Vol. 13). Cambridge University Press.

Libois, Q., Proulx, C., Ivanescu, L., Coursol, L., Pelletier, L. S., Bouzid, Y., ... Blanchet, J.-P. (2016). A microbolometer based far infrared radiometer to study thin ice clouds in the arctic. Atmospheric Measurement Techniques, $9(4), 1817$.

Lupu, C., Gauthier, P., \& Laroche, S. (2011). Evaluation of the impact of observations on analyses in 3d-and 4d-var based on information content. Monthly Weather Review, 139(3), 726-737.

McNally, A., Watts, P., A Smith, J., Engelen, R., Kelly, G., Thépaut, J., \& Matricardi, M. (2006). The assimilation of airs radiance data at ecmwf. Quarterly Journal of the Royal Meteorological Society, 132(616), 935-957.

Merrelli, A., \& Turner, D. D. (2012). Comparing information content of upwelling far-infrared and midinfrared radiance spectra for clear atmosphere profiling. Journal of Atmospheric and Oceanic Technology, 29(4), 510-526.

Mertens, C. J. (2002). Feasibility of retrieving upper tropospheric water vapor from observations of far-infrared radiation. In Optical spectroscopic techniques, remote sensing, and instrumentation for atmospheric and space research iv (Vol. 4485, pp. 191-201).

Mlynczak, M., Johnson, D. G., \& Kratz, D. P. (2007). The far-infrared spectrum: Exploring a new frontier in the remote sensing of the earth's climate. In $\mathrm{Hy}$ perspectral imaging and sounding of the environment (p. HMA1).

Mlynczak, M. G., Harries, J. E., Rizzi, R., Stackhouse, P. W., Kratz, D. P., John- 
son, D. G., ... Soden, B. J. (2002). Far-infrared: a frontier in remote sensing of earth's climate and energy balance. In International symposium on optical science and technology (pp. 150-158).

Mlynczak, M. G., Johnson, D. G., Latvakoski, H., Jucks, K., Watson, M., Kratz, D. P., ... others (2006). First light from the far-infrared spectroscopy of the troposphere (first) instrument. Geophysical research letters, 33(7).

Müller, R., Kunz, A., Hurst, D. F., Rolf, C., Krämer, M., \& Riese, M. (2016). The need for accurate long-term measurements of water vapor in the upper troposphere and lower stratosphere with global coverage. Earth's Future, 4(2), $25-32$.

Palchetti, L., Olivieri, M., Pompei, C., Labate, D., Brindley, H., Di Natale, G., \& Bianchini, G. (2016). The far infrared fts for the forum mission. In Fourier transform spectroscopy (pp. FTu3C-1).

Proulx, C., Williamson, F., Allard, M., Baldenberger, G., Gay, D., Garcia-Blanco, S., ... others (2009). The earthcare broadband radiometer detectors. In Proc. spie (Vol. 7453, p. 74530S).

Purser, R., \& Huang, H. (1993). Estimating effective data density in a satellite retrieval or an objective analysis. Journal of applied meteorology, 32(6), 10921107.

Rabier, F., Fourrié, N., Chafäi, D., \& Prunet, P. (2002). Channel selection methods for infrared atmospheric sounding interferometer radiances. Quarterly Journal of the Royal Meteorological Society, 128(581), 1011-1027.

Rizzi, R., Serio, C., \& Amorati, R. (2002). Sensitivity of broadband and spectral measurements of outgoing radiance to changes in water vapor content. In Optical spectroscopic techniques, remote sensing, and instrumentation for atmospheric and space research iv (Vol. 4485, pp. 181-190).

Rodgers, C. D. (2000). Inverse methods for atmospheric sounding: theory and practice (Vol. 2). World scientific.

Serreze, M. C., Barrett, A. P., \& Stroeve, J. (2012). Recent changes in tropospheric water vapor over the arctic as assessed from radiosondes and atmospheric reanalyses. Journal of Geophysical Research: Atmospheres, 117(D10).

Shahabadi, M. B., \& Huang, Y. (2014). Measuring stratospheric h2o with an airborne spectrometer. Journal of Atmospheric and Oceanic Technology, 31(7), $1502-1515$.

Shahabadi, M. B., Huang, Y., \& Moreau, L. M. (2015). Measuring stratospheric h 2 o with an airborne spectrometer: Simulation with realistic detector characteristics. IEEE Journal of Selected Topics in Applied Earth Observations and Remote Sensing, 8(7), 3541-3545.

Susskind, J., Barnet, C. D., \& Blaisdell, J. M. (2003). Retrieval of atmospheric and surface parameters from airs/amsu/hsb data in the presence of clouds. IEEE Transactions on Geoscience and Remote Sensing, 41(2), 390-409.

Wark, D., \& Hilleary, D. (1969). Atmospheric temperature: Successful test of remote probing. Science, 165(3899), 1256-1258.

Wielicki, B. A., Young, D., Mlynczak, M., Thome, K., Leroy, S., Corliss, J., ... others (2013). Achieving climate change absolute accuracy in orbit. Bulletin of the American Meteorological Society, 94(10), 1519-1539.

Xiong, X., Wenny, B. N., Wu, A., Barnes, W. L., \& Salomonson, V. V. $\quad$ (2008). Aqua modis thermal emissive band on-orbit calibration, characterization, and performance. IEEE Transactions on Geoscience and Remote Sensing, 47(3), 803-814. 